\title{
Impact of dynamical charm quarks
}

\section{$\bar{Z}$ LPHA \\ Collaboration}

\section{Tomasz Korzec*, Francesco Knechtli}

University of Wuppertal, Gaußstr. 20, 42119 Wuppertal, Germany

E-mails: korzec@uni-wuppertal.de, knechtli@uni-wuppertal.de

\section{Salvatore Calì}

University of Wuppertal, Gaußstr. 20, 42119 Wuppertal, Germany and University of Cyprus, P.O. Box 20537, 1678 Nicosia, Cyprus

E-mail: scalieuni-wuppertal.de

\section{Björn Leder}

Humboldt Universität zu Berlin, Newtonstr. 15, 12489 Berlin, Germany

E-mail: lederaphysik.hu-berlin.de

\section{Graham Moir}

Department of Applied Mathematics and Theoretical Physics, Centre for Mathematical Sciences,

University of Cambridge, Wilberforce Road, Cambridge, CB3 OWA, UK

E-mail: graham.moir@damtp.cam.ac.uk

We compute and compare the continuum limits of several quantities in QCD with and without a dynamical charm quark. We consider both low energy quantities, like the hadronic scales $r_{0}$ and $t_{0}$, and high energy quantities, like the charmonium masses.

34th annual International Symposium on Lattice Field Theory

24-30 July 2016

University of Southampton, UK

${ }^{*}$ Speaker. 


\section{Motivation}

Many large scale QCD simulations are carried out in the $2+1$ flavor theory, i.e. with light quarks only $[1,2,3,4,5]$. There are several good reasons for this choice.

1. Lattices that are large enough to accommodate (nearly) physical pions, are usually too coarse to resolve correlation lengths associated with charm quarks. Trying to do so leads to pronounced lattice artifacts $[6,7]$.

2. Each additional quark flavor increases the costs of the simulation significantly. Moreover, the effort of tuning the bare parameters leading to a well-defined chiral trajectory is greatly increased.

3. There is strong evidence that the effect of a dynamical charm quark on low energy quantities requires a very high precision to be resolved $[8,9]$.

On the other hand, charm physics becomes more and more interesting. Experiments like Belle, CLEO and BABAR keep discovering new hidden and open charm-states, many of which are poorly understood. Consequently, a huge effort is made to explain some of these findings from first principles. But how reliable is charm physics on ensembles without a dynamical charm quark?

Our goal is the estimation of the effect of "quenching" the charm quark, on quantities that contain valence charm quarks, e.g. on the charmonium mass spectrum.

\section{QCD with two heavy quarks}

To avoid the usual multi-scale problem, we consider a simplified version of QCD, namely a $S U$ (3) Yang-Mills theory coupled to two degenerate heavy quarks. This allows us to perform simulations in relatively small volumes with very small lattice spacings. As a discretization we use Wilson's plaquette gauge action and a clover improved doublet of twisted mass Wilson fermions. At maximal twist, the clover term with non-perturbatively determined [10] coefficient $c_{\mathrm{sw}}$ is not necessary for $O(a)$ improvement of physical observables. However, it was found that its inclusion reduces the $O\left(a^{2}\right)$ lattice artifacts, see e.g. [11].

At the small lattice spacings of our simulations, critical slowing down is a major obstacle. We use open boundary conditions in the time directions to keep auto-correlation times associated with the topological charge manageable [12]. The boundary improvement coefficients are kept at their tree-level values $c_{G}=1$ and $c_{F}=1$.

The bare coupling was chosen such that the lattice spacings cover the range $0.023 \mathrm{fm} \lesssim a \lesssim$ $0.036 \mathrm{fm}$. The hopping parameter $\kappa$ was set to its critical value in order to achieve maximal twist. The critical values were obtained from an interpolation of published data [13, 14]. The twisted mass parameter $\mu$ was chosen such that the RGI mass in our simulations matches that of a charm quark, more precisely, at a given value of the bare coupling the twisted mass parameter is

$$
a \mu=\frac{M}{\Lambda_{\overline{M S}}} Z_{P}\left(L_{1}\right) \frac{\bar{m}\left(L_{1}\right)}{M} \Lambda_{\overline{M S}} L_{1} \frac{a}{L_{1}},
$$

where we set $\frac{M}{\Lambda_{\overline{M S}}}=4.87$. The pseudo-scalar renormalization constant at renormalization scale $L_{1}^{-1}$ in the Schrödinger Functional scheme, the relation between the running and the RGI mass $\bar{m} / M$ 


\begin{tabular}{cccccccc}
\hline ID & $\frac{T}{a} \times\left(\frac{L}{a}\right)^{3}$ & $\beta$ & $\kappa$ & $a \mu$ & $r_{0} / a$ & $t_{0} / a^{2}$ & MDUs \\
\hline P & $120 \times 32^{3}$ & 5.700 & 0.136698 & 0.113200 & $9.131(56)$ & $9.105(37)$ & 8592 \\
W & $192 \times 48^{3}$ & 6.000 & 0.136335 & 0.072557 & $14.27(15)$ & $22.36(13)$ & 22400 \\
\hline qP & $120 \times 32^{3}$ & 6.340 & $(0.1357769)$ & $(0.11,0.12,0.13)$ & $9.029(80)$ & $9.035(30)$ & 20080 \\
qW & $192 \times 48^{3}$ & 6.672 & $(0.1353155)$ & $(0.07,0.08,0.09)$ & $14.103(94)$ & $21.925(82)$ & 73920 \\
qX & $192 \times 64^{3}$ & 6.900 & $(0.1344651)$ & $(0.054)$ & $18.65(24)$ & $39.43(17)$ & 100000 \\
& & & $(0.1344597)$ & $(0.056)$ & & & \\
& & & $(0.1344540)$ & $(0.058)$ & & & \\
\hline
\end{tabular}

Table 1: Simulation parameters of our ensembles. The columns show the ensemble names, the lattice sizes, the gauge couplings $\beta=6 / g_{0}^{2}$, the hopping parameters (for maximal twist), the twisted mass parameters, the scales $r_{0} / a$ and $t_{0} / a^{2}$ and the total statistics in molecular dynamics units. The quenched simulations $\mathrm{qP}, \mathrm{qW}$ and qX need mass parameters only for the measurements, they are given in parentheses. Three values of $\mu$ were used for the quenched measurements.

and the $\Lambda$ parameter of two flavor QCD in units of $L_{1}$ are known from [13]. To obtain the scale $L_{1}$ in lattice units at a particular value of the bare coupling, short interpolations were necessary. Some of the quantities entering eq. (2.1) have rather large errors. The errors of $Z_{P}$ and of the relative scale change are propagated throughout our calculation, while the other amount to a change in the target value for $M / \Lambda$.

In order to quantify the impact of dynamical charm quarks, we also simulate the pure gauge theory at values of the scales $r_{0} / a, t_{0} / a^{2}$ which are similar or larger. Table 1 summarizes our ensembles.

\section{Strategy}

On the generated ensembles we measure the following quantities:

\subsection{Gradient flow observables}

A gradient flow equation can be solved in order to relate the simulated gauge fields to gauge fields at a fictitious flow-time $t$ [15]. Local operators built from gauge fields at finite flow time do not require renormalization and have variances that remain finite in the continuum limit. One particularly useful flow quantity is the flow scale $t_{0}$ defined by [16]

$$
t_{0}^{2}\left\langle G_{\mu \nu}^{a}\left(t_{0}\right) G_{\mu \nu}^{a}\left(t_{0}\right)\right\rangle=0.3,
$$

where $G_{\mu v}(t)$ is the field strength tensor at flow time $t$. We use a symmetric (clover) discretization of $G_{\mu \nu}$ and the Wilson action in the flow equation, exactly as in [16]. The action density is averaged over the time-slices far away from the temporal boundaries.

\subsection{Wilson loops}

Another set of useful, purely gluonic, observables are the Wilson loops. We follow [17] and measure Wilson loops where the initial and final line of gauge links are smeared using up to four 
levels of HYP smearing. This allows us to extract the static-quark potential $a V(r)$ very reliably by solving a generalized eigenvalue problem [18]. The static force $F(r)=V^{\prime}(r)$ can then be used to measure the hadronic scale $r_{0}$ defined implicitly through $\left.r^{2} F(r)\right|_{r=r_{0}}=1.65$ [19] or a renormalized coupling at scale $r^{-1}$, that can be defined by

$$
\alpha_{q q}\left(r^{-1}\right)=\frac{1}{C_{F}} r^{2} F(r)
$$

\subsection{Meson correlation functions}

In addition to purely gluonic observables, we measure meson correlation functions ${ }^{1}$

$$
\begin{aligned}
C_{\mathscr{O}_{\Gamma}, \mathscr{O}_{\Omega}}\left(x_{0}, y_{0}\right) & =\left\langle\mathscr{O}_{\Gamma}\left(x_{0}\right) \mathscr{O}_{\Omega}^{\dagger}\left(y_{0}\right)\right\rangle \\
\text { with } \quad \mathscr{O}_{\Gamma}\left(x_{0}\right) & =\sum_{\vec{x}} \bar{c}(x) \Gamma c^{\prime}(x), \quad \Gamma \in\left\{\gamma_{5}, \gamma_{0} \gamma_{5}, \gamma_{1}, \gamma_{2}, \gamma_{3}\right\} .
\end{aligned}
$$

Here $c$ and $c^{\prime}$ denote the two flavors in a twisted mass doublet. The measurements involve stochastic time-diluted estimators with $16 U(1)$ noise vectors.

The ground state energy $a m_{\mathscr{O}}$ in a channel determined by the choice of $\mathscr{O}$ is then given by the weighted plateau average of the effective mass

$$
\operatorname{am}_{\mathscr{O}}^{\mathrm{eff}}\left(x_{0}\right)=\log \left[\frac{C_{\mathscr{O} \mathscr{O}}\left(a, x_{0}\right)}{C_{\mathscr{O} \mathscr{O}}\left(a, x_{0}+a\right)}\right] .
$$

Of particular interest will be the pseudo-scalar and vector mass $m_{P}=m_{\mathscr{O}_{\gamma_{5}}}, m_{V}=m_{\mathscr{O}_{\gamma_{1}}}=m_{\mathscr{O}_{\gamma_{2}}}=$ $m_{\mathscr{O}_{\gamma_{3}}}$. Around the charm quark mass both pseudo-scalar and vector correlators can be measured very precisely up to large distances between source and sink. Figure 1 shows the effective masses and the plateaux averages for the case of the qX ensemble.

In addition to the meson masses, we monitor the PCAC mass, to make sure that we are close enough to maximal twist on all ensembles.

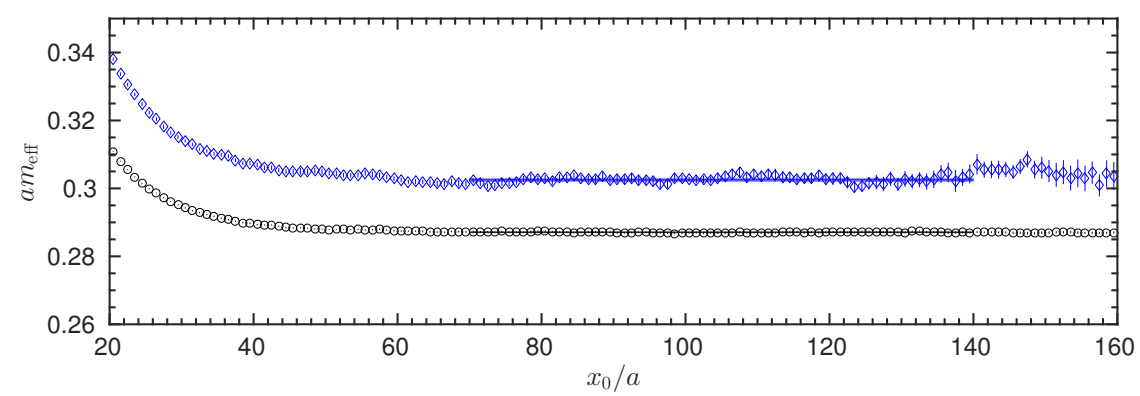

Figure 1: Effective masses and plateaux averages of the ground states in the pseudo-scalar (circles) and the vector (diamonds) channel on the ensemble qX.

\subsection{Quenched measurements}

In the $N_{f}=0$ calculations the mass and twisted mass parameters $\kappa$ and $\mu$ are required only for the computation of observables with valence quarks. The two conditions that fix these bare

\footnotetext{
${ }^{1}$ Given here in the physical basis. In the twisted basis, the vector operators acquire an additional factor $\gamma_{5}$.
} 
parameters are that we want to be at maximal twist $\leftrightarrow m_{P C A C}=0$, and that we want to be at the same pseudo-scalar mass as in the dynamical simulations $\leftrightarrow \sqrt{t_{0}} m_{P}=\left[\sqrt{t_{0}} m_{P}\right]_{\text {cont. }}^{N_{f}=2}$. The critical hopping parameters were obtained from an interpolation of values in [20], where also $c_{\mathrm{sw}}$ was determined non-perturbatively. The measurements were carried out at three values of $\mu$, so that a safe interpolation to the tuning-point could be performed. The PCAC masses turned out to be too large on the qX ensemble, so the parameter $\kappa$ was re-tuned (for each $\mu$ value separately). Figure 2 shows the interpolation procedure on the ensemble qP. The situation is very similar for the two finer lattices. On the quenched ensembles qP, qW and qX the twisted mass parameters $a \mu^{*}$ that lead to $\sqrt{t_{0}} m_{P}=1.816(32)$ are $a \mu^{*}=0.1233(03)(32), a \mu^{*}=0.0781(03)(20)$ and $a \mu^{*}=0.0581(02)(15)$ respectively. The source of the first error is the statistical precision of $\sqrt{t_{0}} m_{P}$ on the quenched ensembles. The second error is due to the uncertainty of the tuning goal.
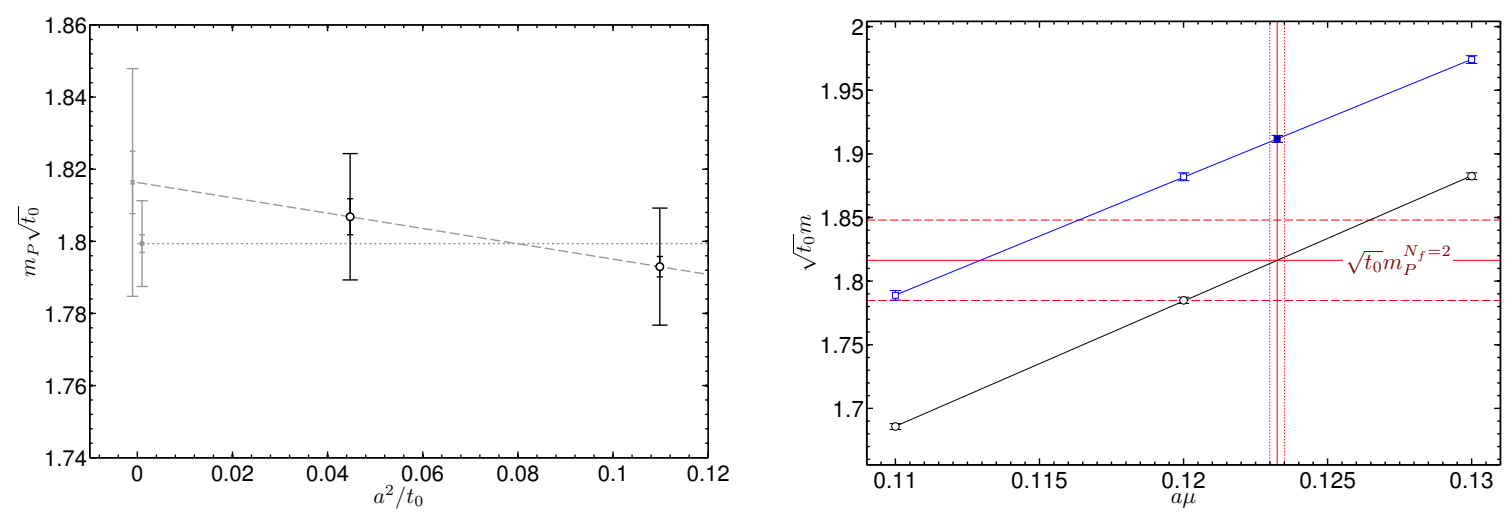

Figure 2: The left panel shows the continuum extrapolation of the pseudo-scalar mass in units of $\sqrt{t_{0}}$ on the $N_{f}=2$ ensembles. Extrapolations both constant and linear in $a^{2}$ are shown. The continuum result from the linear extrapolation is used to define the tuning point for the quenched ensembles. The smaller error bars are statistical errors, while the complete errors contain contributions from uncertainties in $a \mu$. The right panel shows the interpolation of the measured pseudo-scalar masses (open circles) on the $N_{f}=0$ ensemble $q P$. The horizontal lines depict the tuning point and its error. The vertical lines are the resulting interpolated twisted mass parameter $a \mu^{*}$ and its statistical error. The measured vector meson masses (open squares) can then be interpolated to the tuning point, resulting in the solid square point. The depicted error does not contain the uncertainty in $a \mu^{*}$, which however is taken into account in our final results.

\section{Results and conclusions}

In low energy observables we cannot resolve an effect of the dynamical heavy quark. This is shown in figure 3 for the ratio of hadronic scales $\frac{r_{0}}{\sqrt{t_{0}}}$, for which an effect of $\lesssim 0.3 \%$ was predicted in [8]. At our current precision of $1.5 \%$ it would be a surprise to see a significant difference.

Another example is given by $\alpha_{q q}\left(r^{-1}\right)$ at low energies. Here however a difference that cannot be explained by lattice artifacts is observed, once the renormalization scale rises to about $2 \mathrm{GeV}$.

Two cases of observables involving valence charm quarks are shown in figure 4 . The first is the ratio of vector over pseudo-scalar meson mass. This quantity is particularly precise, because the two mesons have a similar dependence on the bare mass and errors due to uncertainties in $a \mu^{*}$ cancel to a large extent. We find that the impact of dynamical charm content in the sea on this 

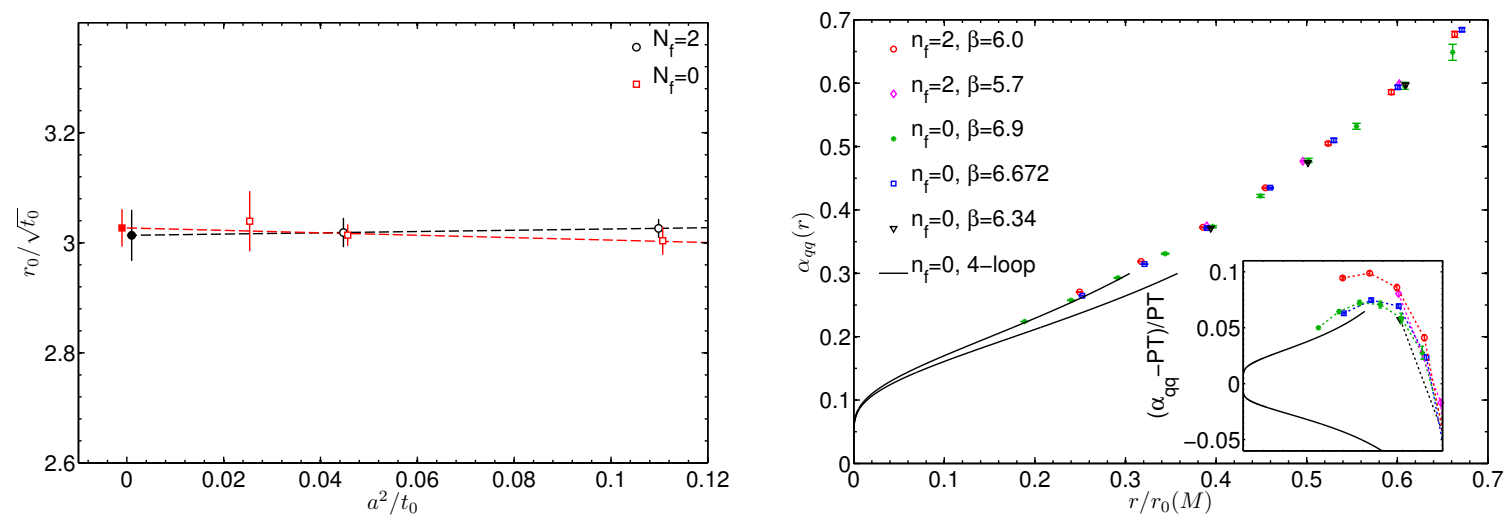

Figure 3: Comparison of purely gluonic observables in $N_{f}=2$ and $N_{f}=0$ theories. In the left panel the continuum extrapolations of a dimensionless ratio of scales in the two theories is shown. The continuum results (solid markers) are compatible with each other. In the right panel the results for the renormalized coupling of eq. (3.2) are shown for all our ensembles. The solid lines stem from $N_{f}=0$ four loop perturbation theory. Their spread is due to the uncertainty in $\Lambda$. The relative deviation from perturbation theory at high energies is shown separately. Here a clear dependence of $\alpha_{q q}$ on the number of flavors can be observed.
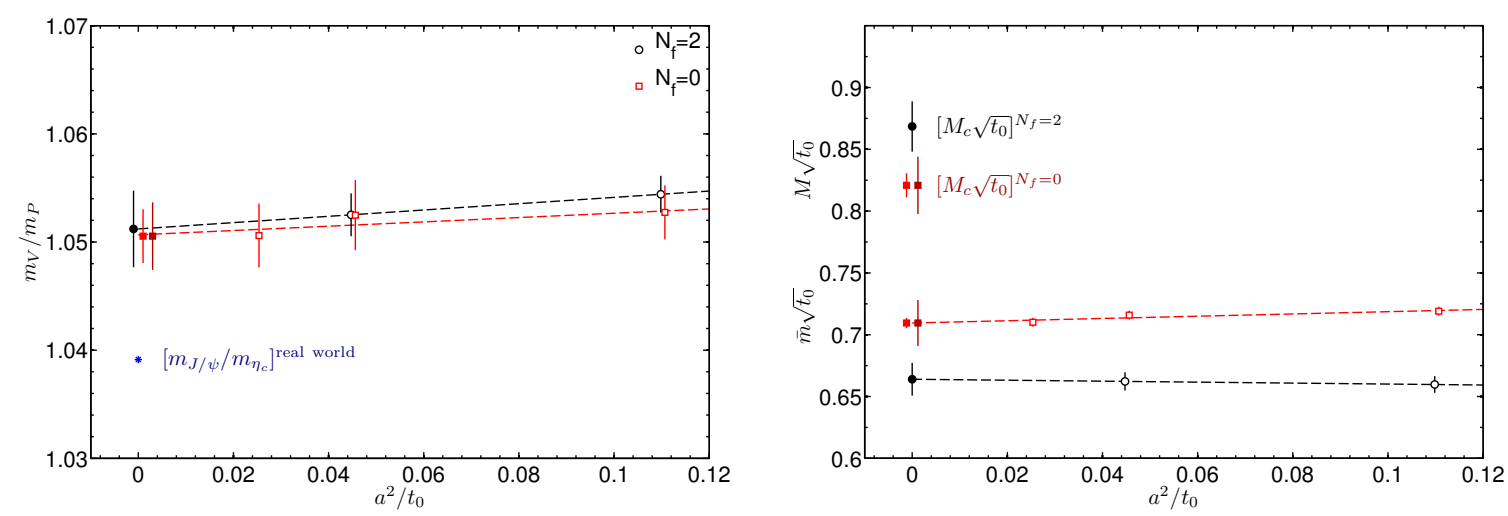

Figure 4: The left panel shows the continuum extrapolation of the ratio of vector to pseudo-scalar meson mass. The continuum extrapolated values with errors are shown. The measured ratio of the corresponding charmed mesons is shown as well. Light sea quarks, disconnected contributions and electromagnetism are presumably responsible for the $1 \%$ deviation to our number. The right panel shows a continuum extrapolation of the running masses. Their continuum values are translated to RGI masses which are shown as well. In the $N_{f}=0$ case, the smaller error neglects uncertainties in $a \mu^{*}$, while the larger includes them.

quantity is tiny and below $0.3 \%$. The second is the RGI quark mass $M_{c}$ in units of $\sqrt{t_{0}}$. We first determine the continuum values of $\bar{m} \sqrt{t_{0}}$. The agreement/disagreement of these continuum values is meaningless, because the running masses $\bar{m}$ are not renormalized at the same scale. However, since the ratios $\bar{m} / M$ are known in the two theories [13,21], both can be translated into a RGI mass, for which a comparison makes sense. We find a deviation between the RGI masses of the two theories of $(5.5 \pm 3.5) \%$. It would be certainly useful to further reduce the error on this number.

\section{Acknowledgments}

We thank Rainer Sommer and Jochen Heitger for many fruitful discussions and Martin Lüscher 
and Stefan Schaefer for making openQCD [22] available. We gratefully acknowledge the computer resources granted by the Gauss Centre for Supercomputing (GCS) through the John von Neumann Institute for Computing (NIC) on the GCS share of the supercomputer JUQUEEN at JSC, with funding by the German Federal Ministry of Education and Research (BMBF) and the German State Ministries for Research of Baden-Württemberg (MWK), Bayern (StMWFK) and NordrheinWestfalen (MIWF). We are further grateful for computer time allocated for our project on the Konrad and Gottfried computers at the North-German Supercomputing Alliance HLRN. This project has received funding from the European Union's Horizon 2020 research and innovation programme under the Marie Sklodowska-Curie grant agreement No 642069. G.M. acknowledges support from the Herchel Smith Fund at the University of Cambridge.

\section{References}

[1] H.-W. Lin et. al. Phys. Rev. D79 (2009) 034502.

[2] S. Aoki et. al. Phys. Rev. D81 (2010) 074503.

[3] W. Bietenholz et. al. Phys. Lett. B690 (2010) 436-441.

[4] R. Arthur et. al. Phys. Rev. D87 (2013) 094514.

[5] M. Bruno et. al. JHEP 02 (2015) 043.

[6] Y. Cho, S. Hashimoto, A. Jüttner, T. Kaneko, M. Marinkovic, J.-I. Noaki and J. T. Tsang JHEP 05 (2015) 072.

[7] T. D. Rae and S. Dürr PoS LATTICE2015 (2016) 094.

[8] M. Bruno, J. Finkenrath, F. Knechtli, B. Leder and R. Sommer Phys. Rev. Lett. 114 (2015), no. 10 102001.

[9] F. Knechtli, M. Bruno, J. Finkenrath, B. Leder and R. Sommer PoS LATTICE2015 (2016) 256.

[10] K. Jansen and R. Sommer Nucl. Phys. Proc. Suppl. 63 (1998) 853-855.

[11] P. Dimopoulos, H. Simma and A. Vladikas JHEP 07 (2009) 007.

[12] M. Lüscher and S. Schaefer JHEP 07 (2011) 036.

[13] P. Fritzsch, F. Knechtli, B. Leder, M. Marinkovic, S. Schaefer, R. Sommer and F. Virotta Nucl. Phys. B865 (2012) 397-429.

[14] P. Fritzsch, N. Garron and J. Heitger JHEP 01 (2016) 093.

[15] M. F. Atiyah and R. Bott Phil. Trans. Roy. Soc. Lond. A308 (1982) 523-615.

[16] M. Lüscher JHEP 08 (2010) 071. [Erratum: JHEP03,092(2014)].

[17] M. Donnellan, F. Knechtli, B. Leder and R. Sommer Nucl. Phys. B849 (2011) 45-63.

[18] B. Blossier, M. Della Morte, G. von Hippel, T. Mendes and R. Sommer JHEP 04 (2009) 094.

[19] R. Sommer Nucl. Phys. B411 (1994) 839-854.

[20] M. Lüscher, S. Sint, R. Sommer, P. Weisz and U. Wolff Nucl. Phys. B491 (1997) 323-343.

[21] A. Jüttner, Precision lattice computations in the heavy quark sector. $\mathrm{PhD}$ thesis, Humboldt U., Berlin, 2004.

[22] M. Lüscher and S. Schaefer Comput. Phys. Commun. 184 (2013) 519-528. 